\title{
Eficácia da terapia manual
no tratamento das disfunções
temporomandibulares temporomandibulares
}

Effectiveness of manual

therapy in the treatment of

FisiSenectus. Unochapecó Ano 7, n. 2 - Jul/Dez. 2019 temporomandibular disorders p. $53-66$

Nicolly Márcia Nunes da Silva.nicollymnunes@hotmail.com

Fisioterapeuta pela Faculdade de Integração do Sertão (FIS) - Serra Talhada (PE).

Luciana Ângelo Bezerra.naninhabezerra@yahoo.com.br

Fisioterapeuta pela Faculdade Integrada do Recife, mestre em Ciências da Saúde pela Universidade Federal de Pernambuco, doutora em Neuropsiquiatria e Ciências do Comportamento pela Universidade Federal de Pernambuco subárea neurociências, professora do Curso de Fisioterapia da Faculdade de Integração do Sertão (FIS) - Serra Talhada (PE).

\section{Nylene Maria Rodrigues da Silva. nylenersb@gmail.com}

Fisioterapeuta pelas Faculdades Integradas de Patos (PB), especialista em Fisioterapia na UTI pela PulmocárdioInterfisio Recife (PE), conveniada com a Faculdade Redentor (RJ), professora especialista do Curso de Fisioterapia da Faculdade de Integração do Sertão (FIS) - Serra Talhada (PE).

\section{Resumo}

Introdução: a disfunção temporomandibular (DTM) é um termo que se aplica a um grupo de desordens funcionais da ATM e estruturas mastigatórias, que apresenta etiologia complexa e multifatorial. Objetivo: avaliar a eficácia da Terapia Manual (TM) sobre a sintomatologia das disfunções temporomandibulares (DTMs). Materiais e métodos: foram selecionados seis voluntários de ambos os sexos com diagnóstico prévio de distúrbio cinético-funcional da articulação temporomandibular (ATM), que responderam ao Questionário da Academia Americana de Dor Orofacial para triagem inicial. Os voluntários foram submetidos a uma avaliação fisioterapêutica por meio de um questionário semiestruturado para observar aspectos funcionais e motores, além de sinais e sintomas de DTM, e houve a aplicação da Escala Visual Analógica (EVA) para dor; quanto à observância do grau de DTM, foi utilizado o Índice Anamnésico de Fonseca e Índice de Disfunção Clínica Craniomandibular (IDCCM) com Índice de Mobilidade Mandibular (IMM). Após a comprovação do diagnóstico, os voluntários foram submetidos à aplicação de um protocolo fisioterapêutico que compreendeu dez sessões de TM. Em seguida, foram reavaliados e a condição sintomática e funcional foi abordada em análise comparativa, através do software SPSS 17.0, ANOVA, com o teste t de student, para análise da variância da condição pré e pós-tratamento com TM. Resultados e Conclusão: os resultados obtidos mostraram que houve significância estatística, sendo $(p<0,05)$ no nível de dor, sintomatologia, grau de disfunção e aumento da mobilidade mandibular. Observou-se que a terapia manual apresentou eficácia sobre o tratamento das DTMs.

\section{Palavras-chave}

Articulação Temporomandibular. Síndrome da Disfunção da Articulação Temporomandibular. Dor Orofacial. Fisioterapia. 


\begin{abstract}
Introduction: the temporomandibular dysfunction (TMD) is a term that applies to a group of TMJ functional disorders and masticatory structures, which has a complex and multifactorial etiology. Objective: to evaluate the efficacy of Manual Therapy (MT) on the symptomatology of temporomandibular disorders (TMDs). Materials and Methods: six volunteers of both genres with a previous diagnosis of functional kinetic disturbance of the temporomandibular joint (TMJ) who answered the questionnaire of the American Academy of Orofacial Pain for initial screening. They were submitted to a physical therapy evaluation through a semistructured questionnaire to observe functional and motor aspects, as well as signs and symptoms of TMD and the application of Analog Visual Scale (AVS) for pain; Regarding the degree of TMD grade, the Fonseca Anamnestic Index and Craniomandibular Clinical Dysfunction Index (CMCDI) with Mandibular Mobility Index (MMI) were used. After the diagnosis was confirmed the volunteers were submitted to the application of a physiotherapeutic protocol that comprised (10) TM sessions. Afterwards, they were reassessed and the symptomatic and functional condition were addressed in a comparative analysis, through the SPSS 17.0 Software, ANOVA, with the Student's t-test, to analyze the variance of the condition pre- and post-treatment with TM. Results and conclusion: The results showed that there was statistical significance being $(p<0.05)$ in the level of pain, symptomatology, degree of dysfunction and increased mandibular mobility. It was observed that manual therapy showed efficacy on TMD treatment.
\end{abstract}

\title{
Keywords
}

Temporomandibular joint; Temporomandibular Joint Dysfunction Syndrome; Orofacial Pain; Physiotherapy.

\section{Introdução}

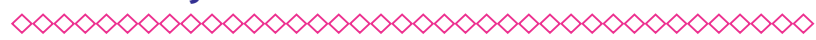

A articulação temporomandibular (ATM) é a única articulação móvel do crânio que está localizada bilateralmente à frente das orelhas e na extremidade posterossuperior da mandíbula. É a articulação que liga a fossa mandibular do osso temporal, superiormente (parte fixa) e à cabeça da mandíbula; inferiormente (parte móvel), essas extremidades ósseas estão envolvidas por uma cápsula articular, separadas por um disco articular que fornece amortecimento durante os movimentos, estabilizadas pelo ligamento lateral e ligamento medial.Os ligamentos estilomandibular e esfenomandibular influenciam somente na cinemática, além do líquido sinovial, que tem a função de lubrificar a articulação e possibilitar seu movimento normal e sem comprometimento $0^{1,2}$.

Sendo considerada uma das articulações mais complexas e mais utilizadas do nosso corpo, a ATM realiza movimento de dobradiça e deslizamento, 0 que a classifica como uma articulação ginglimoartrodial. Ela é responsável pelos movimentos de depressão da mandíbula (abertura da boca), elevação da mandíbula (fechamento da boca), desvio lateral (movimento lateral da mandíbula), protrusão (movimento anterior da mandíbula) e retração (movimento posterior da mandíbula) executados pelos músculos mastigatórios, o que possibilita 0 ato de mastigar, deglutir, bocejar, falar, entre outras atividades ${ }^{1}$.

Portanto quaisquer desequilíbrios neuromusculares e alterações nas estruturas articulares podem causar deslocamentos uni ou bilaterais dos discos articulares e disfunção do sistema mastigatório e articular, afetando diretamente a funcionalidade dessa articulação, prejudicando o seu desempenho e resultando em uma disfunção da mesma ${ }^{3}$.

As Disfunções Temporomandibulares (DTMs) têm etiologia complexa e multifatorial, associada a fatores predisponentes, iniciadores e perpetuantes, relacionados com alterações oclusais, falta de dentes, lesões traumáticas ou degenerativas das doenças sistêmicas, transtorno interno do disco articular e das demais estruturas que compõem a articulação, problemas esqueléticos, disfunções musculares, hipomobilidade ou hipermobilidade da articulação e disfunção das articulações da cervical, além de fatores psicológicos e hábitos deletérios ou parafuncionais ${ }^{4,5}$.

São caracterizadas por sinais e sintomas que incluem dor ou desconforto na ATM; dor facial; dor na musculatura mastigatória e cervical, apresentando-se uni ou bilateralmente; cefaleia, otalgias, 
zumbido nos ouvidos; estalidos; crepitação; amplitude de movimento mandibular limitada; desvios na mandíbula e consequentes desvios posturais; dificuldade de mastigação e de realização de outras atividades que envolvem os movimentos mandibulares ${ }^{6,7}$.

0 tratamento da DTM tem uma abordagem interdisciplinar, que depende da estreita relação entre a intervenção de cada profissional que compõe a equipe e uma abordagem individualizada para cada paciente, pois cada um vai apresentar causas e características específicas, graus e severidade de sinais e sintomas diferentes, mesmo havendo a mesma disfunção ${ }^{6}$.

O fisioterapeuta possui como técnica principal no tratamento das DTMs a terapia manual, que corresponde a um conjunto de manipulações e mobilizações articulares, liberação miofascial, pompage, alongamento dos tecidos moles, com o objetivo de estimular a propriocepção, produzir elasticidade a fibras aderidas, estimular a produção e a distribuição do líquido sinovial, melhorar a nutrição da fibrocartilagem da superfície articular da ATM, possibilitar a correção de falhas posicionais dos côndilos, recuperar a força dos músculos envolvidos na dinâmica da ATM, proporcionar relaxamento muscular, controle do quadro álgico e ganho do arco de movimento ${ }^{4,8}$.

Contudo o objetivo do estudo foi avaliar a eficácia da terapia manual (TM) sobre a sintomatologia das DTMs, pois a fisioterapia tem sido considerada um tratamento alternativo para a meIhora da sintomatologia de pacientes com DTM, caracterizando-se como uma terapia simples, não invasiva e de baixo custo se comparada a outros tratamentos. Apesar de existirem várias propostas de tratamento fisioterapêutico para esse quadro clínico, optou-se pelo uso da TM, visto que a literatura apresenta poucos dados científicos relevantes que confirmem os efeitos e a eficácia da técnica.

\section{Materiais e Métodos}

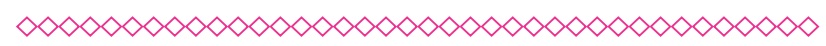

Trata-se de um estudo descritivo correlacional e experimental de abordagem quantitativa, demonstrando comparações entre variáveis nos momentos pré e pós-tratamento ${ }^{9}$. Foi aprovado pelo Comitê de Ética em Pesquisa com Seres Humanos da Faculdade de Integração do Sertão (FIS) de acordo com o parecer de número 3.279.907.

0 estudo foi realizado em uma instituição de ensino superior (IES) privada, localizada no município de Serra Talhada/PE, no período de abril a maio de 2019. Os voluntários foram selecionados através de triagem utilizando o Questionário da Academia Americana de Dor Orofacial ${ }^{10}$. A amostra foi constituída por seis indivíduos de ambos os sexos previamente diagnosticados com distúrbio cinético-funcional da ATM com idade entre 19 e 51 anos, cujos critérios de inclusão foram: não estar fazendo uso de aparelho ortodôntico, não ter nem estar realizando tratamento fisioterapêutico nem acompanhamento fonoaudiológico para o tratamento da DTM, além daqueles que compreendessem e executassem comandos verbais simples. Foram estabelecidos como critérios de exclusão indivíduos que faziam uso de prótese dentária móvel, que possuíam deformidade congênita da ATM e que estavam em tratamento medicamentoso para DTM.

Ao concordarem em participar do estudo, assinaram o Termo de Consentimento Livre e Esclarecido (TCLE), obedecendo às Resoluções $n^{\circ} 466 / 12$ e 510/16, da Comissão Nacional de Ética em Pesquisa/Conselho Nacional de Saúde (Conep/CNS). Em seguida, foram agendadas avaliações individuais, na Clínica Escola de Fisioterapia da IES, com duração média de uma hora cada.

Para avaliar os aspectos funcionais e motores da ATM, foi aplicado um questionário semiestruturado $^{11}$, que possibilitou traçar o perfil biodemográfico da amostra, avaliar a condição de saúde e o histórico relacionado à disfunção da ATM, bem como os hábitos de vida; além do exame físico e do diagnóstico cinético-funcional. Foi utilizada a fotogrametria digital com a finalidade de se verificar a postura através do software $\mathrm{SAPO}^{\circledR}$ e identificar possíveis alterações e/ou desvios posturais nos seguintes seguimentos: cabeça, cervical e ombros. 0 estetoscópio Rappaport Premium ${ }^{\circledR}$ foi utilizado para realizar ausculta da ATM e o martelo de reflexo Buck MD Neurológico $18 \mathrm{~cm}$ foi utilizado para testar o reflexo mentoniano.

A Escala Visual Analógica (EVA) para dor foi inserida no questionário semiestruturado para 
avaliação fisioterapêutica da ATM, para auxiliar na quantificação da intensidade da dor no voluntário. Para avaliar a severidade dos sinais e sintomas das DTMs, foram aplicados questionários em forma de entrevistas sem que houvesse interferências nas respostas, tais como o Questionário e Índice Anamnésico de Fonseca ${ }^{12}$, cuja somatória dos pontos atribuídos resulta em um índice anamnésico que permite classificar os voluntários em categorias de severidade de sintomas: sem DTM (0 a 15 pontos), DTM leve (20 a 45 pontos), DTM moderada (50 a 65 pontos) e DTM severa (70 a 100 pontos).

Já o Índice de Disfunção Clínica Craniomandibular (Clinical DysfunctionIndex, ou Índice de Helkimo (IDCCM) ${ }^{13}$ com Índice de Mobilidade Mandibular (IMM) (tradução não oficial) avalia os voluntários em quatro categorias: sem sintomas de DTM (0 ponto), sintomas leves (1 a 4 pontos), moderados ( 5 a 9 pontos) e severos (10 a 25 pontos). Os dados necessários para preencher o item "a" do índice clínico foram obtidos pelo IMM, que é usado para classificar a mobilidade mandibular em normal (0 ponto), ligeiramente reduzida (1 a 4 pontos) e severamente reduzida (5 a 20 pontos).

Após a avaliação, foram agendadas as sessões conforme compatibilidade de horários dos entrevistados, atendendo também à disponibilidade da Clínica Escola de Fisioterapia da IES. 0 tratamento proposto envolvia a aplicação de um protocolo fisioterapêutico que compreendia 10 sessões de TM, com frequência de duas vezes por semana, com duração de 50 minutos cada sessão ${ }^{14}$. Estas foram supervisionadas pela professora orientadora.

Os atendimentos foram realizados de forma individual e em sala fechada. 0 estado inicial e final dos voluntários a cada atendimento foi avaliado por meio da verificação dos sinais vitais, assim como eram questionados sobre a presença de dor e a localização da mesma.

As técnicas de TM realizadas foram: mobilização dos tecidos moles (músculos temporal, masseter superficial e profundo, piterigoideo medial e lateral, supra-hioideos, esternocleidomastoideo, escalenos, elevadores da escápula, trapézio superior, médio e inferior, suboccipitais); mobilização da coluna cervical (tração, manipulação com flexão, manipulação com rotação); manipulação e mobilização da ATM (tração, deslizamento ventral, deslizamento medial, mobilização com balanceio); e condicionamento muscular com alongamento passivo dos músculos da ATM, cervical e cintura escapular e exercícios de resistência aos movimentos de abertura, fechamento, lateralidade e protrusão da mandíbula repetidos por 10 vezes ${ }^{15,16}$.

Finalizada a aplicação do protocolo fisioterapêutico, os pacientes foram reavaliados utilizando os mesmos instrumentos da avaliação inicial e a condição sintomática e funcional foi abordada em análise comparativa. Os dados foram analisados através do software Microsoft Excel 2010 para cálculos que incluem média, mínima, máxima e desvio padrão, além de valores percentuais e do teste $t$ de student pareado, através do SPSS 17.0, ANOVA, para análise da variância da condição pré e pós-tratamento com TM. Os resultados foram expressos em gráficos e tabelas.

\section{Resultados}

$\infty<\infty<\infty<\infty<\infty<\infty<\infty<\infty<\infty<\infty<\infty<\infty<\infty<\infty<\infty<\infty<\infty$

Ao analisar a amostra, observou-se que o sexo predominante foi o feminino. Quanto ao IMC, os entrevistados apresentaram média de $24,98 \mathrm{~kg} / \mathrm{m}^{2}$, estando dentro dos limites de normalidade segundo o Ministério da Saúde; a amostra foi composta principalmente por estudantes, conforme apresentado na Tabela 1.

A Tabela 2 aponta o estilo de vida e as condições de saúde dos entrevistados. Observou-se que $5(83,33 \%)$ indivíduos da amostra apresentaram outras afecções diagnosticadas e apenas 2 (33,33\%) fizeram uso de medicação para as mesmas; $33,33 \%$ praticavam exercício físico de forma regular; $100 \%$ da amostra relatou estar em contato frequente com situações estressantes e obteve média de sono de 5,33 horas por noite, o que está abaixo do recomendado para adultos jovens.

A Tabela 3 apresenta os resultados referentes à condição geral da ATM pré e pós-tratamento. Quanto à presença de sinais e sintomas, os mais frequentes foram a dor articular, o estalido articular e a crepitação; em relação aos fatores que agravam a dor, os mais citados foram durante a mastigação e condições estressantes, assim como 
maior dificuldade para mastigar; na ausculta da ATM, observou-se a presença de crepitação bilateral e estalido unilateral; observaram-se também dois casos de subluxação da ATM. Quanto à severidade dos sinais e sintomas e à classificação da disfunção, tanto no Índice Anamnésico de Fonseca quanto no IDCCM, foi observada prevalência de disfunção severa; após o tratamento, a amostra apresentou grau de disfunção moderado; no IMM, também foi observada melhora dos movimentos mandibulares. Os testes estatísticos demonstraram que houve diferenças estatisticamente significativas, sendo $(p<0,05)$ quando comparados os valores pré e pós-tratamento.

A partir do Gráfico 1, é possível observar que os voluntários apresentavam mais de um sintoma associado à dor na ATM. Os mais frequentes foram a dor nos dentes, cefaleia, cervicalgia e dor no ouvido.

0 Gráfico 2 apresenta os resultados referentes à presença de alterações posturais nos seguimentos: cabeça, cervical e ombros; as alterações mais importantes observadas foram a protrusão ou anteriorização da cabeça e hiperlordose cervical.

\section{Discussão}

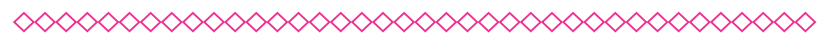

Quanto ao sexo dos indivíduos, a amostra foi composta por seis voluntários, sendo cinco $(83,33 \%)$ do sexo feminino e um $(16,66 \%)$ do sexo masculino, corroborando estudos que apontam as mulheres como sendo mais acometidas com disfunções na ATM que os homens, em uma proporção de cinco mulheres para cada homem ${ }^{17,18}$. Segundo Nekora-Azak ${ }^{19}$, o padrão de início da patologia após a puberdade e a redução das taxas de prevalência no período pós-menopausa sugerem que os hormônios reprodutivos possam ter papel importante na etiologia da DTM. Okeson ${ }^{20}$ e Bove et $\mathrm{al}^{17}$ relatam que a maior concentração de indivíduos com DTM tem idade entre 21 e 30 anos. Os dados obtidos neste estudo também mostram média de idade de 25,83 anos.

A amostra apresentou média de 24,98 no Índice de Massa Corpórea (IMC), considerado valor limite dentro dos valores de normalidade para adultos jovens. Tal valor pode ser justificado pelo fato de $66,66 \%$ da amostra não praticar exercício físico de maneira regular. Os voluntários relataram estar em contato frequente com situações estressantes e apresentaram média de sono de 5,33 horas por dia, fatores estes que influenciam na sintomatologia da disfunção temporomandibular. Segundo Alves Junior ${ }^{21}$, recomendam-se pelo menos oito horas de sono por dia; o mesmo autor afirma que, durante o sono, o organismo regula o sistema imunológico e o sistema hormonal e recompõe os neurotransmissores, além de determinar o sucesso diurno, pois melhora o humor, a energia, a produtividade e a saúde.

Observou-se que a maior queixa dos voluntários investigados foi a dor na ATM, relatada por $100 \%$ dos voluntários, sendo agravada por mastigação prolongada em $83,33 \%$ dos casos e condições estressantes vivenciadas no dia a dia em $50 \%$ dos casos. Os valores pré e pós-aplicação da Escala Visual Analógica (EVA) para dor demonstraram diferenças estatisticamente significativas, sendo $(p<0,05)$, pois a terapia manual produz um efeito inibitório da dor, reduzindo a sensibilização dos mecanorreceptores, produzidos a partir das fibras aferentes, tendo como hipótese que a analgesia imediata induzida manualmente pode estar relacionada a um bloqueio sensorial, com a diminuição dos impulsos nervosos periféricos para o Sistema Nervoso Central (SNC) ${ }^{22}$.

A dor na ATM apresenta uma alta incidência nas disfunções temporomandibulares, além de grande quantidade de sinais e sintomas associados ${ }^{17,20,23}$. No presente estudo, corroborando as pesquisas citadas, o motivo da adesão ao tratamento foi a dor; os voluntários também apresentavam mais de um sinal ou sintoma associado, sendo que os principais foram dor nos dentes, cefaleia, cervicalgia e dor no ouvido. Após o tratamento, houve diminuição relevante na quantidade de pacientes que apresentavam um ou mais sinais e sintomas associados à dor.

A limitação de mobilidade mandibular é considerada um dos principais sinais clínicos da DTM. Em adultos, a média do limite máximo da abertura mandibular encontra-se entre 53 e $58 \mathrm{~mm}$, podendo variar de 40 a $60 \mathrm{~mm}^{24}$. Neste estudo, observou-se que a maioria dos voluntários apresentava abertura máxima menor do que o valor limite de normalidade. Os testes demonstraram que não 
houve diferenças estatisticamente significativas, sendo $(p>0,05)$ quando comparados os valores pré e pós-tratamento. No estudo de Dworkin e LeResche ${ }^{25}$, a maioria dos pacientes estudados apresentava restrição da abertura máxima voluntária; foi utilizada a medida de $35 \mathrm{~mm}$ de abertura como referência de normalidade.

Já o IMM visto de maneira isolada para avaliar os movimentos de depressão, elevação, desvio lateral e protrusão da mandíbula comprovou que houve melhora significativa em todos os movimentos, sendo $(p<0,05)$ quando comparados os valores pré e pós-tratamento.

Sobre as condições funcionais na ATM, qualquer alteração na articulação, e dependendo da capacidade adaptativa do indivíduo, poderão ocorrer manifestações e desconforto clínico, gerando deficiências funcionais no sistema estomatognático como um todo, por meio de sinais e sintomas, que limitam ou incapacitam as atividades fisiológicas do indivíduo ${ }^{11}$. Neste estudo, observou-se que $83,33 \%$ da amostra apresentou dificuldade para mastigar e 33,33\% para bocejar e beijar. Após a aplicação do protocolo fisioterapêutico, apenas $16,66 \%$ da amostra apresentou dificuldade para mastigar e para beijar. Tal resultado comprova que as técnicas de condicionamento muscular aplicadas melhoraram a funcionalidade e aumentaram a resistência à fadiga. Observou-se ainda que $33,33 \%$ da amostra permaneceu com dificuldade para bocejar, devido à limitação na abertura bucal, que apresentou uma melhora, porém não ocorreu estatística significante.

Segundo Okeson ${ }^{15}$, uma ATM que apresenta configuração anatômica de sua eminência articular em declive posterior curto e inclinado seguido de um declive anterior mais longo tende a subluxar. Clinicamente, avalia-se a subluxação observando a abertura máxima da boca; caso esteja presente, no último estágio da abertura, o côndilo "pula" para a frente, deixando uma pequena depressão atrás de $\mathrm{si}^{26}$. No presente estudo, $16,66 \%$ da amostra apresentou subluxação à direita e $16,66 \%$ à esquerda, com os sinais clínicos descritos pela literatura; após o tratamento e reavaliação, observou-se meIhora, uma vez que o fortalecimento dos músculos e ligamentos que compõem a ATM objetiva a estabilização articular através do aumento do trofismo de forma equilibrada, pois a subluxação ocorre quando há presença de fraqueza da musculatura envolvida nos movimentos da ATM.

Os hábitos parafuncionais podem causar alterações nas funções musculoesqueléticas, alterando o fluxo sanguíneo, gerando dor e alterando a posição da mandíbula em relação ao crânio e à região cervical ${ }^{17,23}$, além de acarretar abrasões dentárias severas, destruição de trabalhos odontológicos, dores de cabeça tensionais, alterações auditivas, degeneração dos tecidos articulares, hipertrofia muscular severa, entre outras manifestações ${ }^{27}$. Neste estudo, os dados encontrados em relação aos hábitos parafuncionais, tais como onicofagia (roer unhas), apertamento dos dentes e mordida da própria boca, evidenciam a hiperatividade dos músculos mastigatórios, um dos fatores que predispõem à ocorrência da disfunção na ATM.

Sendo os músculos da mastigação sinérgicos aos da cervical, um desequilíbrio entre eles causa forças retrusivas na mandíbula, alterando o seu posicionamento de repouso e levando a hiperatividade muscular ${ }^{28}$. A atividade aumentada da musculatura mastigatória interfere nos músculos de contra apoio (esternocleideomastoide e trapézio), que leva a um encurtamento dos músculos posteriores do pescoço e alongamento dos anteriores. Essa posição anteriorizada da cabeça pode levar a um distúrbio de posicionamento e funcionamento mandibular, que pode gerar tensão na musculatura mastigatória, agravando a sintomatologia da DTM ${ }^{29}$. Os dados obtidos neste estudo também mostram que $100 \%$ da amostra apresentou anteriorização da cabeça.

Ao realizar a anteriorização da cabeça, o olhar tende a ficar baixo e, na tentativa de nivelá-lo, tornando-o funcional, ocorre o aumento da lordose cervical, refletindo na musculatura da mastigação ${ }^{30}$. Neste estudo, pôde-se observar que $66,66 \%$ da amostra apresentou hiperlordose cervical.

As técnicas de terapia manual possibilitam motivar a capacidade de reparo do organismo, uma vez que trabalham nas propriedades dos tecidos moles, como músculos, meniscos, tendões e ligamentos, que, quando submetidos à terapia manual, tendem a ter aumento da vascularização local e diminuição de rigidez, fatos estes que concorrem para a diminuição da dor e melhora do desempenho funcional dos músculos que se 
inserem na ATM e, consequentemente, na mobilidade mandibular ${ }^{22}$. Depois de submetidos à terapia manual, os voluntários que participaram deste estudo apresentaram uma melhora considerável no que concerne à diminuição do quadro doloroso e dos sintomas associados, do grau de disfunção e da mobilidade da articulação temporomandibular.

\section{Conclusão}

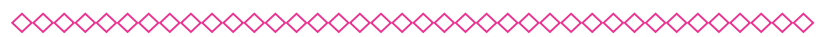

Os resultados obtidos neste estudo apontam a complexidade da DTM, o que a torna multifatorial, visto que sofre influência de diferentes fatores e apresenta uma variedade de sinais e sintomas. Contudo foi observado que a terapia manual apresenta eficácia no tratamento das DTMs, pois houve significância estatística na condição sintomática e funcional, quando comparados os valores pré e pós-tratamento.

A terapia manual é um dos recursos fisioterapêuticos mais importantes para o tratamento de pacientes com DTM, porém poucos estudos têm investigado os efeitos dessa intervenção terapêutica de maneira isolada. Por isso, recomenda-se a realização de estudos longitudinais com maiores amostras e número de sessões, para aprofundar o conhecimento sobre a eficácia da terapia manual no tratamento das disfunções que acometem a ATM e sobre a atuação da fisioterapia no público acometido por tal afeç̧ão.

Nota: artigo científico apresentado ao curso de Bacharelado em Fisioterapia da Faculdade de Integração do Sertão (FIS) - Serra Talhada (PE) como requisito para obtenção de grau de Bacharel em Fisioterapia (2019).

\section{Referências}

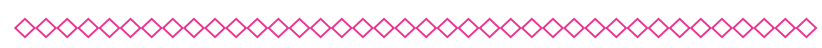

1. Lippert L. Cinesiologia Clínica e Anatomia. Rio de Janeiro: Guanabara Koogan; 2013.

2. Paulsen F, Waschke J. Sobotta: atlas de anatomia humana: cabeça, pescoço e neuroanatomia. Rio de Janeiro: Guanabara Koogan; 2015.

3. Macedo PFA, Bianchini EMG. Myofunctional orofacial examination: comparative analysis in young adults with and without complaints. CoDAS. Socied Brasil de Fono. 2014;26(6):464-70.

4. Oliveira KB, Pinheiro ICO, Freitas DG, Gualberto HD, Carvalho NA. A abordagem fisioterapêutica na disfunção da articulação temporomandibular. Revisão da literatura. Med Reabil. 2010;29(3):61-4.

5. Ferreira IMMS. Tratamento fisioterapêutico das disfunções da articulação temporomandibular: uma revisão bibliográfica. 2015 .

6. Torres F, Campos LG, Fillipini HF, Weigert $\mathrm{KL}$, Dalla Vecchia GF. Efeitos dos tratamentos fisioterapêuticos e odontológicos em pacientes com disfunção temporomandibular. Fisioter. Mov. 2012;25(1):117-25.

7. Costa ET, Nascimento LAO, Fernandes KJM. Toxina botulínica no tratamento de disfunção temporomandibular miofascial: revisão de literatura. RevAcBO. 2018;27(1):96-102.

8. Barbosa VCS, Barbosa FS. Fisioterapia nas Disfunções Temporomandibulares. São Paulo: Phorte; 2009.

9. Marconi MA; Lakatos EM. Fundamentos de metodologia científica. São Paulo: Atlas; 2010.

10. Okeson JP. Dor orofacial: guia de avaliação, diagnóstico e tratamento. São Paulo: Quintessence; 1998.

11. Salvatti SC. Terapia manual integrada e reeducação e reeducação funcional dos distúrbios temporomandibulares de origem muscular. 2012.

12. Fonseca $D M$, Bonfate $G$, Valle $A L$, Freitas SFT. Diagnóstico pela anamnese da disfunção craniomandibular. Rev Gaucha Odontol. 1994;42:23-8.

13. Helkimo M. Studies on function and dysfunction of the masticatory system, II: index for anamnestic and clinical dysfunction and occlusal state. Sven Tandlak Tidskr. 1974;67(2):101-21. 
14. Silva MN, Barbosa VCS, Barbosa FS. Estudo intervencional de pacientes portadores de disfunções temporomandibulares submetidos ao acompanhamento fisioterapêutico. Rev cient Faminas. 2016;5(1):52-61.

15. Okeson JP. Tratamento das desordens temporomandibulares e oclusão. Elsevier Brasil; 2008.

16. Edmond SL; Shionis L; Harrington C. Manipulação e mobilização: técnicas para membros e coluna. São Paulo: Manole, 2000.

17. Bove SRK, Guimarães AS, Smith RL. Caracterização dos pacientes de um ambulatório de disfunção temporomandibular e dor orofacial. Rev Latino-am Enferm. 2005;13(5):686-91.

18. Biasotto-Gonzalez DA. Articulação temporomandibular. In: Biasotto-Gonzalez DA, organizador. Abordagem interdisciplinar das disfunções temporomandibulares. Barueri: Manole; 2005.

19. Nekora-Azak A. Temporomandibular disorders in relation to female reproductive hormones: a literature review. J Prosthet Dent. 2004;91(5):492-3.

20. Okenson JP. Fundamentos de oclusão e desordens temporomandibular. Artes Médicas: São Paulo; 2000.

21. Alves Júnior DR. Repercussão do sono sobre o trabalho. Diagn Tratamento. 2010;15(3):150-2.

22. Ruaro JA. Análise da adequação de técnicas de terapia manual de cotovelo e punho no tratamento da síndrome do túnel do carpo: estudo de caso. Rev Ter Man. 2003;1(4):106-12.
23. Pereira KNF, Andrade LLS, Costa MLG, Portal TF. Sinais e sintomas de pacientes com disfunção temporomandibular. Rev CEFAC. 2005;7(2):221-8.

24. Dworkin SF, Huggins KH, LeResche L, Von KM, Howard J, Truelove E. Epidemiology of signs and symptoms in temporomandibular disorders: clinical signs in cases and controls. J Am Dent Assoc. 1990;120:81-273.

25. Dworkin SF, LeResche L. Research diagnostic criteria for temporomandibular disorders: review, criteria, examinations and specifications, critique. J Craniomandib Disord. 1992;6:301-55.

26. Bernardino Júnior R, Teixeira M, Goulart LV, Guedes LI, Costa EMC. Avaliação de Técnica Alternativa Aplicada ao Tratamento Imediato de Luxação Espontânea da Articulação Têmporo Mandibular. Biosci. J. 2006;22(3):105-11.

27. Maciel RN. ATM e dores craniofaciais: fisiopatologia básica. São Paulo: Santos; 2003.

28. Yi LC, Guedes ZCF, Vieira MM. Relação da postura corporal com a disfunção da articulação temporomandibular: Hiperatividade dos músculos da mastigação. Fisioterapia Brasil. 2003;4(5):341-7.

29. Arenhante R, Lazarotto $R$, Thomé K. Tratamento fisioterapêutico na disfunção temporomandibular: um estudo de caso. Rev FisiSenectus. 2013;1:109-17.

30. Burgess JA, Sommers EE, Truelove EL, Dworkin SF. Efeitos a curto prazo de dois métodos terapêuticos na dor miofascial e deficiência orgânica do sistema mastigatório. Dente de J Prosthet. 1998;60:606-10. 


\section{Anexos}

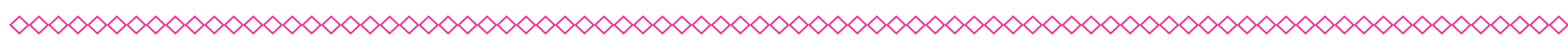

Tabela 1 - Características sociodemográficas e antropométricas da amostra

\begin{tabular}{|c|c|c|c|c|}
\hline Variáveis & Categorias & $\begin{array}{c}\text { GE } \\
n(06)\end{array}$ & $\%$ & \\
\hline \multirow[t]{2}{*}{ Sexo } & Masculino & 01 & 16,66 & \\
\hline & Feminino & 05 & 83,33 & \\
\hline \multirow[t]{2}{*}{ Estado civil } & Casado & 01 & 16,66 & \\
\hline & Solteiro & 05 & 83,33 & \\
\hline \multirow[t]{3}{*}{ Ocupação atual } & $\begin{array}{l}\text { Estudantes } \\
\text { Secretaria }\end{array}$ & $\begin{array}{l}05 \\
01\end{array}$ & $\begin{array}{l}83,33 \\
16,66\end{array}$ & \\
\hline & \multicolumn{3}{|c|}{ Amostra n (06) } & \\
\hline & Média & Máxima & Mínima & $\mathrm{Dp}$ \\
\hline Idade (anos) & 25,83 & 51 & 19 & 12,48 \\
\hline $\mathrm{IMC}\left(\mathrm{kg} / \mathrm{m}^{2}\right)$ & 24,98 & 31,1 & 16,6 & 4,96 \\
\hline
\end{tabular}

GE - Grupo Experimental; n - número absoluto; Dp - Desvio padrão; IMC - Índice de Massa Corpórea; kg/m² - Quilograma por metro quadrado.

Fonte: Dados da pesquisa (2019).

(clique para voltar ao texto) 
Tabela 2 - Estilo de vida e condições de saúde

\begin{tabular}{|c|c|c|c|c|}
\hline Variáveis & Categorias & $\begin{array}{c}\text { GE } \\
n(06)\end{array}$ & $\%$ & \\
\hline \multirow{3}{*}{ Afecções diagnosticadas } & Distimia & 01 & 16,66 & \\
\hline & Hipertensão & 01 & 16,66 & \\
\hline & $\begin{array}{l}\text { Osteomioarticulares em } \\
\text { outras regiões do corpo }\end{array}$ & 03 & 50 & \\
\hline Faz uso de medicação & SIM & 02 & 33,33 & \\
\hline para as mesmas & NÃO & 04 & 66,66 & \\
\hline Pratica exercício & SIM & 02 & 33,33 & \\
\hline de forma regular & NÃO & 04 & 66,66 & \\
\hline \multirow[t]{3}{*}{$\begin{array}{l}\text { Tem contato frequente com } \\
\text { situações estressantes }\end{array}$} & SIM & 06 & 100 & \\
\hline & & $\begin{array}{l}\text { Amostra n } \\
(06)\end{array}$ & & \\
\hline & Média & Máxima & Mínima & $\mathrm{Dp}$ \\
\hline Sono (horas) & 5,33 & 6 & 5 & 0,51 \\
\hline
\end{tabular}

GE - Grupo Experimental; n - número absoluto; Dp - Desvio padrão.

Fonte: Dados da pesquisa (2019).

(clique para voltar ao texto) 
Tabela 3 - Caracterização da amostra quanto à condição sintomática e funcional da DTM pré e pós-tratamento com TM

\begin{tabular}{|c|c|c|c|c|c|}
\hline Variáveis & Categorias & $\begin{array}{c}\text { GE } \\
n(06)\end{array}$ & $\begin{array}{l}\text { PRÉ } \\
\%\end{array}$ & $\begin{array}{c}\text { GE } \\
n(06)\end{array}$ & $\begin{array}{c}\text { Pós } \\
\%\end{array}$ \\
\hline $\begin{array}{l}\text { História anterior de } \\
\text { disfunção na ATM }\end{array}$ & $\begin{array}{l}\text { SIM } \\
\text { NÃO }\end{array}$ & $\begin{array}{l}03 \\
03\end{array}$ & $\begin{array}{l}50 \\
50\end{array}$ & $\begin{array}{l}03 \\
03\end{array}$ & $\begin{array}{l}50 \\
50\end{array}$ \\
\hline Realizou tratamento & NÃO & 06 & 100 & 06 & 100 \\
\hline $\begin{array}{l}\text { Presença de um } \\
\text { ou mais sinais e } \\
\text { sintomas na ATM }\end{array}$ & $\begin{array}{l}\text { Dor articular } \\
\text { Crepitação } \\
\text { Bloqueio articular } \\
\text { Estalido articular } \\
\text { Sobressalto }\end{array}$ & $\begin{array}{l}06 \\
03 \\
02 \\
04 \\
02\end{array}$ & $\begin{array}{c}100 \\
50 \\
33,33 \\
66,66 \\
33,33\end{array}$ & $\begin{array}{l}03 \\
01 \\
00 \\
02 \\
00\end{array}$ & $\begin{array}{c}50 \\
16,66 \\
0,00 \\
33,33 \\
0,00\end{array}$ \\
\hline $\begin{array}{l}\text { Presença de um ou } \\
\text { mais fatores que } \\
\text { agravam a dor }\end{array}$ & $\begin{array}{l}\text { Condição estressante } \\
\text { Durante a mastigação } \\
\text { Hábitos parafuncionais } \\
\text { Dormir sobre a ATM }\end{array}$ & $\begin{array}{l}03 \\
05 \\
02 \\
02\end{array}$ & $\begin{array}{c}50 \\
83,33 \\
33,33 \\
33,33\end{array}$ & $\begin{array}{l}03 \\
03 \\
01 \\
00\end{array}$ & $\begin{array}{c}50 \\
50 \\
16,66 \\
0,00\end{array}$ \\
\hline $\begin{array}{l}\text { Presença de uma ou } \\
\text { mais dificuldades } \\
\text { funcionais da ATM }\end{array}$ & $\begin{array}{l}\text { Mastigar } \\
\text { Bocejar } \\
\text { Beijar }\end{array}$ & $\begin{array}{l}05 \\
02 \\
02\end{array}$ & $\begin{array}{l}83,33 \\
33,33 \\
33,33\end{array}$ & $\begin{array}{l}01 \\
02 \\
01\end{array}$ & $\begin{array}{l}16,66 \\
33,33 \\
16,66\end{array}$ \\
\hline Ausculta da ATM & $\begin{array}{l}\text { Crepitação unilateral } \\
\text { Crepitação bilateral } \\
\text { Estalido unilateral } \\
\text { Estalido bilateral }\end{array}$ & $\begin{array}{l}02 \\
03 \\
03 \\
02\end{array}$ & $\begin{array}{l}33,33 \\
50 \\
50 \\
33,33\end{array}$ & $\begin{array}{l}03 \\
00 \\
04 \\
01\end{array}$ & $\begin{array}{c}50 \\
0,00 \\
66,66 \\
16,66\end{array}$ \\
\hline $\begin{array}{c}\text { Presença de } \\
\text { subluxação na ATM }\end{array}$ & $\begin{array}{c}\text { À direita } \\
\text { À esquerda }\end{array}$ & $\begin{array}{l}01 \\
01\end{array}$ & $\begin{array}{l}16,66 \\
16,66\end{array}$ & $\begin{array}{l}00 \\
00\end{array}$ & $\begin{array}{l}0,00 \\
0,00\end{array}$ \\
\hline $\begin{array}{l}\text { Presença de um } \\
\text { ou mais hábitos } \\
\text { parafuncionais }\end{array}$ & $\begin{array}{l}\text { Roer unhas } \\
\text { Apertar os dentes } \\
\text { Morder a própria boca } \\
\text { Morder objetos }\end{array}$ & $\begin{array}{l}03 \\
02 \\
04 \\
02\end{array}$ & $\begin{array}{c}50 \\
33,33 \\
66,66 \\
33,33\end{array}$ & $\begin{array}{l}02 \\
02 \\
04 \\
01\end{array}$ & $\begin{array}{l}33,33 \\
33,33 \\
66,66 \\
16,66\end{array}$ \\
\hline $\begin{array}{l}\text { Classificação da } \\
\text { disfunção pelo } \\
\text { Índice de Fonseca }\end{array}$ & $\begin{array}{l}\text { Leve } \\
\text { Moderada } \\
\text { Severa }\end{array}$ & $\begin{array}{l}00 \\
01 \\
05\end{array}$ & $\begin{array}{c}0,00 \\
16,66 \\
83,33\end{array}$ & $\begin{array}{l}02 \\
04 \\
00\end{array}$ & $\begin{array}{c}33,33 \\
66,66 \\
0,00\end{array}$ \\
\hline $\begin{array}{l}\text { Classificação da } \\
\text { disfunção pelo } \\
\text { IDCCM com IMM }\end{array}$ & $\begin{array}{l}\text { Leve } \\
\text { Moderada } \\
\text { Severa }\end{array}$ & $\begin{array}{l}00 \\
00 \\
06\end{array}$ & $\begin{array}{l}0,00 \\
0,00 \\
100\end{array}$ & $\begin{array}{l}01 \\
05 \\
00\end{array}$ & $\begin{array}{c}16,66 \\
83,33 \\
0,00\end{array}$ \\
\hline
\end{tabular}




\begin{tabular}{cccc} 
Variáveis & PRÉ & PÓs & Teste $t$ \\
EVA & M & & $0,01^{*}$ \\
Máx. abertura bucal & 4,33 & 1,5 & 0,19 \\
Índice de Fonseca & 34,84 & 39,14 & $0,008^{*}$ \\
IDCCM & 75 & 51,66 & $0,0002^{*}$ \\
IMM & 16,16 & 7,16 & $0,02^{*}$ \\
\hline
\end{tabular}

DTM - Disfunção Temporomandibular; TM - Terapia Manual; GE - Grupo Experimental; $\mathrm{n}$ - número absoluto; IDCCM Índice de Disfunção Clínica Craniomandibular; IMM - Índice de Mobilidade Mandibular; M - Média; EVA - Escala Visual Analógica.

*As variáveis apresentaram estatística significante $(p<0,05)$.

Fonte: Dados da pesquisa (2019). 
Gráfıco 1 - Presença de um ou mais sintomas associados à dor na ATM

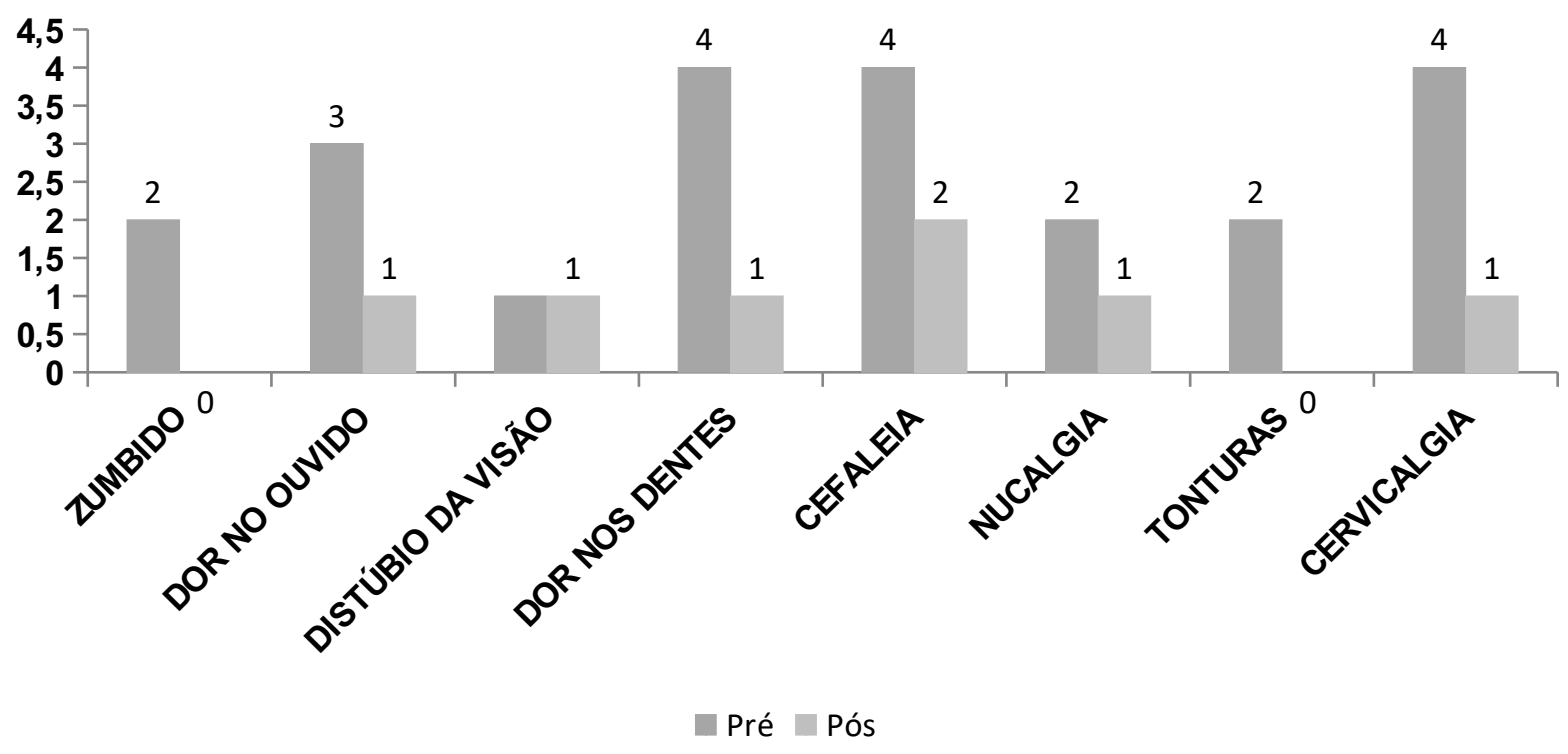

Fonte: Dados da pesquisa (2019).

(clique para voltar ao texto) 
Gráfico 2 - Presença de alterações posturais

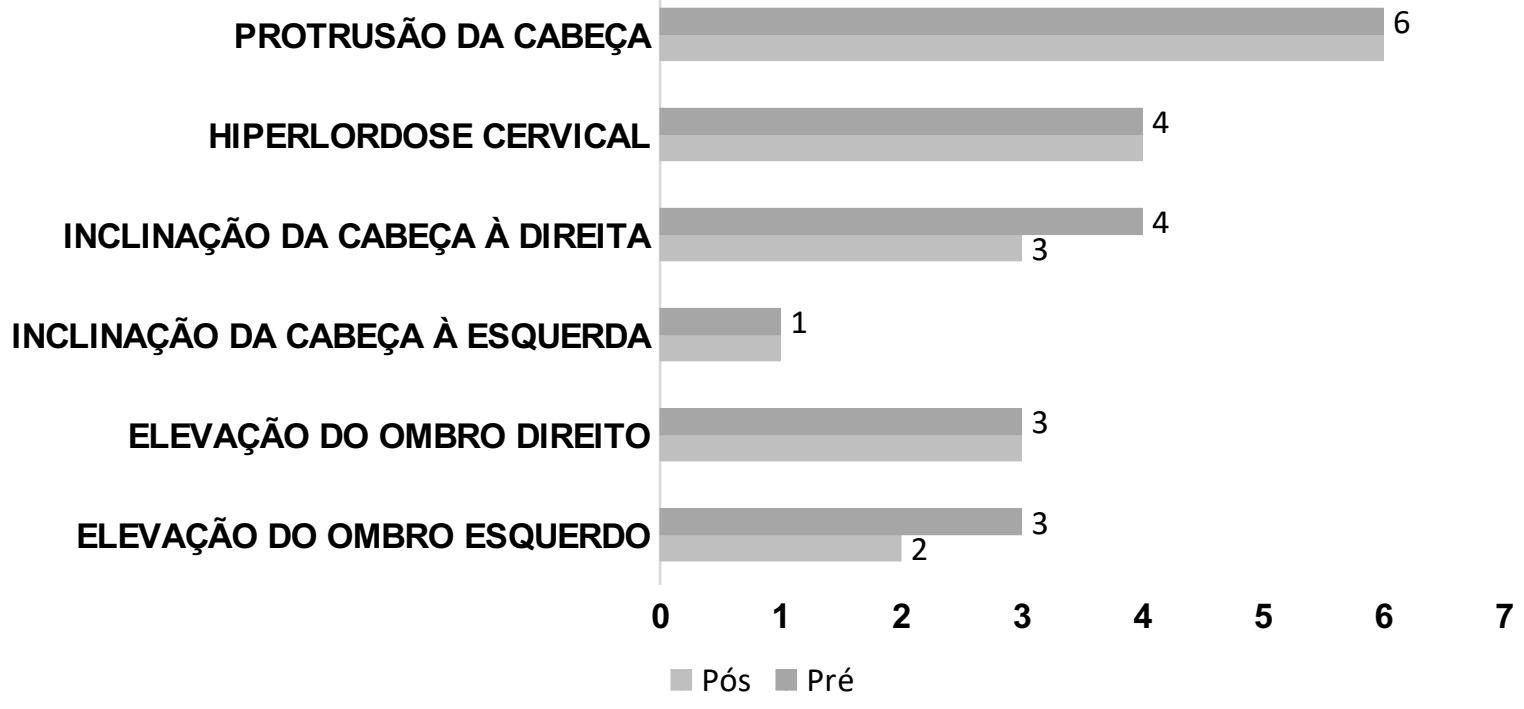

Fonte: Dados da pesquisa (2019). 